VAND-TH-92-5

NSF-ITP-92-77

May 1992

(Revised September 1992)

\title{
Wormhole Cosmology and the Horizon Problem
}

\author{
David Hochberg \\ Department of Physics and Astronomy, Vanderbilt University \\ Nashville, TN 37235, USA \\ and \\ Thomas W. Kephart \\ Department of Physics and Astronomy, Vanderbilt University \\ Nashville, TN 37235, USA and Institute for Theoretical Physics \\ University of California, Santa Barbara, CA 93106, USA
}

\begin{abstract}
We construct an explicit class of dynamic lorentzian wormholes connecting FriedmannRobertson-Walker (FRW) spacetimes. These wormholes can allow two-way transmission of signals between spatially separated regions of spacetime and could permit such regions to come into thermal contact. The cosmology of a network of early Universe wormholes is discussed.
\end{abstract}


The most convincing evidence for the large-scale smoothness of the observable Universe is provided by the cosmic microwave background (CMB) radiation, which was in thermal equilibrium with matter until the Universe cooled to a temperature of about $4000^{\circ} \mathrm{K}$, when the radiation decoupled from matter. From its discovery in 1965, numerous measurements have established the CMB to be consistent with a blackbody spectrum at a temperature $T=2.735 \pm 0.06^{\circ} \mathrm{K}$, and uniform in all directions (on angular scales from $10^{\prime \prime}$ to $180^{\circ}$ ) to about a part in $10^{4}$ [1]. Since this radiation is received from regions which could not have been in causal contact at the time of last scattering, the uniformity in temperature evident in the CMB must be arranged as an initial condition in the big-bang model [2]. This shortcoming of the original big-bang model is known as the horizon problem. There are two ways to solve this problem. One is to postulate that the cosmic scale factor underwent an era of exponential expansion (around 50 e-foldings) such that all the presently observable Universe is in one inflated causal domain. This is the inflationary Universe scenario which has been exhaustively explored in the literature [3]. The second possibility is that the Universe did not necessarily inflate, but has (or had) traversable bridges (wormholes) connecting otherwise causally disconnected regions of spacetime. This Universe with "handles" could alter and perhaps solve the horizon problem. Since this approach has not been studied, we are led to investigate it here, where we envision a network of traversable wormholes existing for a very brief time in the early Universe, arising out of Planck-time quantum metric fluctuations.

Provided wormholes are important for cosmology, then those connecting homogeneous and isotropic spacetimes are undoubtedly the most relevant ones for study (in contradistinction to astrophysical applications, where one expects Schwarzschild [4] or Kerr wormholes to be the most important). Thus, the first step in any investigation of wormhole "phenomenology," and a main result of this work, is the construction of specific cosmological wormhole solutions to Einstein's equations. The wormholes we will consider here result from surgically modified FRW spacetimes. We adopt this technique for convenience's sake only; however, we assume the qualitative features of the wormholes are independent of the details of the construction. To construct them, take two copies of the FRW solution [5] (adopting units where $c=G=1$ )

$$
d s^{2}=-d t^{2}+R^{2}(t)\left(\frac{d r^{2}}{1-\kappa r^{2}}+r^{2}\left(d \theta^{2}+\sin ^{2} \theta d \phi^{2}\right)\right),
$$


and remove from each an identical four-dimensional region of the form $\Omega_{1,2}=\left\{r_{1,2} \leq a\right\}$. The resulting spacetime contains two disjoint boundaries $\partial \Omega_{1,2}=\left\{r_{1,2}=a\right\}$, which are timelike hypersurfaces. An orientation preserving identification $\partial \Omega_{1} \equiv \partial \Omega_{2}$ yields two FRW spacetimes connected by a wormhole whose throat is located on their mutual boundary $\partial \Omega$ [4]. Here, $a=a(\tau)$ is a function describing the dynamics of the wormhole's throat; the physical radius of the wormhole is equal to $a R$. The wormhole is spherically symmetric and the boundary layer is just the world-volume swept out by its throat. This procedure also leads to a wormhole connecting a single FRW spacetime to itself if one identifies the two background spacetimes, i.e., we have a FRW space with a handle. In this case, the two regions $\Omega_{1,2}$ can be separated by an arbitrarily large distance in an open Universe. To insure the modified spacetime is itself a solution of the gravitational field equations requires a proper matching of the metric across the boundary layer. The required junction conditions are most conveniently derived by introducing gaussian normal coordinates in the neighborhood of the boundary hypersurface and integrating the Einstein equations across the boundary [6]. The most general stress-energy tensor which gives rise to two identical FRW spaces attached by a wormhole as described here, is given by

$$
T_{\nu}^{\mu}(x)=S_{\nu}^{\mu} \delta(\eta)+T_{\nu}^{(+)^{\mu}} \Theta(\eta)+T_{\nu}^{(-)^{\mu}} \Theta(-\eta)
$$

where $T_{\nu}^{(+)^{\mu}}=T_{\nu}^{(-)^{\mu}}$ is one of the standard perfect-fluid source terms leading to (1) and

$$
S_{\nu}^{\mu} \equiv \lim _{\epsilon \rightarrow 0} \int_{-\epsilon}^{\epsilon} d \eta T_{\nu}^{\mu}(x)
$$

is the surface stress-energy. The gaussian coordinate $\eta$ parametrizes the proper distance measured perpendicularly through $\partial \Omega$. Integrating $G_{\nu}^{\mu}=8 \pi T_{\nu}^{\mu}$ across $\partial \Omega$ and taking the limit as indicated in (3) yields $[6] S_{\eta}^{\eta}=S_{j}^{\eta}=0$, and

$$
\left(\Delta \mathcal{K}_{j}^{i}-\delta_{j}^{i} \operatorname{Tr}(\Delta \mathcal{K})\right)=8 \pi S_{j}^{i}
$$

where $\Delta \mathcal{K}_{j}^{i} \equiv \lim _{\epsilon \rightarrow 0}\left(\mathcal{K}^{(+)}{ }_{j}^{i}-\mathcal{K}^{(-)_{j}^{i}}\right)$ is the "jump" in the extrinsic curvature of the hypersurface in going from the $-\epsilon$ to the $+\epsilon$ "side". For the case at hand, reflection symmetry implies $\Delta \mathcal{K}_{j}^{i}=2 \mathcal{K}^{(+)^{i}}{ }_{j}$ and the spherical symmetry implies $\mathcal{K}_{j}^{i}=\operatorname{diag}\left(\mathcal{K}_{\tau}^{\tau}, \mathcal{K}_{\theta}^{\theta}, \mathcal{K}_{\theta}^{\theta}\right)$ and $S_{j}^{i}=\operatorname{diag}(-\sigma, P, P)$, where $\sigma, P$ denote the surface energy and pressure densities. The 
extrinsic curvature (in any coordinate system) is defined by

$$
\mathcal{K}_{i, j}=n_{\mu} \nabla_{(j)} \mathrm{e}_{(i)}^{\mu},
$$

where the $\mathrm{e}_{(i)}$ constitute a set of three independent tangent vectors defined along the intrinsic coordinates $\xi^{i}$ parametrizing the hypersurface, and $n^{\mu}$ is the outward unit normal $\left(n_{\mu} n^{\mu}=+1\right.$, for timelike surfaces); the background covariant derivative is taken along the $j^{\text {th }}$ coordinate direction. The throat proper time and the two angles provide a convenient set of intrinsic coordinates: $\xi^{i}=(\tau, \theta, \phi)$. Since the position of the throat (i.e., its embedding in the background FRW space) is $X^{\mu}=(t, a(t), \theta, \phi)$, the tangent vectors are simply $\mathrm{e}_{(i)}^{\mu}=d X^{\mu} / d \xi^{i}$, and the unit normal is $n^{\mu}=\left(\frac{a^{\prime} R}{\left(1-\kappa a^{2}\right)^{1 / 2}}, \frac{1}{R}\left(1-\kappa a^{2}+\left(a^{\prime} R\right)^{2}\right)^{1 / 2}, 0,0\right)$. A straightforward calculation of (5) gives

$$
\mathcal{K}_{\theta}^{\theta}=a^{\prime} \dot{R}+\frac{1}{a R}\left(1+\left(a^{\prime} R\right)^{2}\right)^{1 / 2}
$$

and

$$
\mathcal{K}_{\tau}^{\tau}=\frac{a^{\prime \prime} R}{\left(1+\left(a^{\prime} R\right)^{2}\right)^{1 / 2}}+2 a^{\prime} \dot{R},
$$

where $a^{\prime}=d a / d \tau$ and $\dot{R}=d R / d t[7]$. We have set $\kappa=0$ since the curvature term in (1) is negligible during the early stages of expansion. Note the appearance of the two time parameters $\tau$ and $t$ in (6) and (7). We can always eliminate one in favor of the other (e.g., coordinate time $t)$ by using $a^{\prime}=\dot{a}(d t / d \tau)$ and

$$
(d \tau / d t)=\left(1-(\dot{a} R)^{2}\right)^{1 / 2}
$$

Physically, this amounts to calibrating the clocks attached to the throat in terms of the the comoving clocks. Doing so, and substituting (6) and (7) into (4) yields the wormhole's equations of motion:

$$
-2 \pi \sigma=\frac{ \pm \dot{a} \dot{R}+(a R)^{-1}}{\left(1-(\dot{a} R)^{2}\right)^{1 / 2}}
$$

and

$$
4 \pi P=\frac{ \pm \ddot{a} R \pm 3 \dot{a} \dot{R}+(a R)^{-1}}{\left(1-(\dot{a} R)^{2}\right)^{1 / 2}} \pm \frac{(\dot{a} R)^{2}[\ddot{a} R+\dot{a} \dot{R}]}{\left(1-(\dot{a} R)^{2}\right)^{3 / 2}} .
$$

The plus (minus) sign corresponds to expanding (collapsing) wormholes. We consider examples of both sign choices below. The usual redundancy between the Einstein equations and the conservation of stress-energy $T_{\nu}^{\mu}$, leads to a similar relation between the wormhole 
structure equations (9a) and (9b) and the surface stress tensor (3). The jump in the field equation $G_{j}^{\eta}=8 \pi T_{j}^{\eta}$ together with (4) imply [6]

$$
\nabla_{m} S^{m j}=T^{(-)^{\eta j}}-T^{(+)^{\eta j}}=0
$$

where $\nabla_{m}$ denotes the covariant derivative intrinsic to the hypersurface. Thus, the surface stress-energy tensor is conserved. The derivation of (9a-b) together with (10) constitutes the most important formal result of this Letter.

With the wormhole equations of motion at hand, we now proceed to solve them. As a first example, consider the comoving case, $\dot{a}=a^{\prime}=0$. Then

$$
\sigma=-\frac{1}{2 \pi a R}
$$

and $P=-\frac{1}{2} \sigma$. With a constant throat function $a$, the wormhole's radius simply grows in direct proportion to the background scale factor $R$. This behavior is also confirmed by (8), which implies $t=\tau$. These two time scales coincide if and only if the wormhole throat is coupled to the expansion. The surface energy density is negative, as indeed it must be: the violation of the weak energy condition (WEC) at the wormhole throat is expected on general grounds. In simple terms, the spacetime region surrounding the throat acts as a diverging lens which defocusses light and particle geodesics as they traverse the wormhole. This path divergence reflects a gravitational repulsion at the throat and signals the presence there of a localized negative energy density. Although no classical stress energy tensor is known to violate the WEC, quantum fields in curved backgrounds tend to develop localized negative energy densities $[9,10]$, and suggests these wormholes are driven by quantum field fluctuations, their back-reaction on the metric [11] and by the quantum fluctuations in the metric, as envisioned, for example, in the spacetime foam [12]. According to (11), the comoving wormholes can exist for any value of the fixed throat function $a$. The magnitude of the surface energy density decreases for increasing scale factors. While these comoving wormholes do solve the structure equations, it may be more likely for FRW wormholes to evolve in time in such a way that they decouple from the expansion. Thus we are led to consider decoupled dynamic wormholes, where the full nonlinearity of the structure equations (9a-b) comes into play. 
The strategy for solving the general time dependent case involves selecting a relevant scale factor $R$, a surface energy density $\sigma$ and an equation of state $P=P(\sigma)$ subject to (10). First let us assume a power law expansion $R(t)=R_{i}\left(\frac{t}{t_{i}}\right)^{p}$. Then the choice for $a(t)$ that most simplifies (9a) and (9b) is $a(t)=a_{i}\left(\frac{t}{t_{i}}\right)^{1-p}$ which renders all the denominators constant. Here $R_{i}$ and $a_{i}$ are the scale factor and throat function at some initial time $t_{i}$. Now both $P$ and $\sigma$ go like $t^{-1}$ and the equation of state is in general nonlinear. The relevant power law for early Universe applications is $p=1 / 2$, i.e., a radiation dominated expansion. For this case the surface energy density is $\sigma=\epsilon_{0} / a^{2}$, where $\epsilon_{0}$ is a constant with units of mass given below. Then the exact solution of (9a) and (9b) has

$$
a(t)=a_{i}\left(\frac{t}{t_{i}}\right)^{1 / 2}
$$

and an equation of state as given below. Note that the wormhole throat function is related to the background expansion: $\dot{a} / a=\dot{R} / R$. Moreover, the wormhole motion is decoupled from the expansion since $\frac{1}{a R} \frac{d(a R)}{d t}>\frac{\dot{R}}{R}$. The connection between proper and cosmic time implied by (8) is $t=$ const. $\times \tau$, where the constant of proportionality is greater than one, and depends on $a_{i}, R_{i}$ and $t_{i}$.

For each $t_{i}$, there is an upper bound on the size spectrum of initial wormhole throat radii which follows if we assume no point on the throat can move faster than the speed of light [13]: $|\beta|<1$ where $\beta \equiv 2 \dot{a} R / c$ is a constant for the class of solutions treated here (with $p=1 / 2$ ). For radiation dominated expansion, this condition implies

$$
a_{i} R\left(t_{i}\right) \leq(a R)_{\max } \equiv c t_{i}
$$

where $R\left(t_{i}\right)$ is the scale factor for radiation dominance evaluated at some initial time $t_{i}$ [14]. The surface energy density is given by (restoring $c$ and $G$ )

$$
\epsilon_{0}=-\frac{c a_{i}{ }^{2}}{4 \pi G t_{i}}\left(\frac{\beta+\beta^{-1}}{\left(1-\beta^{2}\right)^{1 / 2}}\right)
$$

and is negative, as for the comoving case.

The equation of state for the $p=1 / 2$ case is given by

$$
P=-\frac{\sigma}{2}\left(\frac{1 \pm 2 \beta^{2}}{1 \pm \beta^{2}}\right)
$$


with sign choices as in $(9 \mathrm{a})$ and $(9 \mathrm{~b})$. Note specifically that in the $\beta \rightarrow 0$ limit $P=$ $-\sigma / 2$ which corresponds to comoving wormholes in agreement with (11) and the subsequent discussion. Other interesting limits are $\beta=1$ for expanding wormholes where $P=-3 \sigma / 4$; while for collapsing wormholes, $\beta=1 / \sqrt{2}$ yields $P=0$, corresponding to pressureless negative energy "dust"; and $\beta \rightarrow 1$ where $P$ diverges.

Thus far, we have dealt with aspects of a single comoving or decoupled wormhole in a FRW background. It is clear, however, that the above construction can be applied an indefinite number of times and will lead to an explicit solution of Einstein's equation with an arbitrary number of wormholes. In fact, this construction is an application of the connected sum operation, familiar from algebraic topology. In general, we let $\mathcal{M}_{1}$ and $\mathcal{M}_{2}$ be two spacetimes and remove from each the interiors of the four-dimensional discs $D_{1} \subset \mathcal{M}_{1}$ and $D_{2} \subset \mathcal{M}_{2}$, and then identify the boundary sets up to homeomorphism $h: \partial D_{1} \rightarrow \partial D_{2}$. The resulting manifold defines the connected sum of $\mathcal{M}_{1}$ and $\mathcal{M}_{2}: \mathcal{M}_{1} \# \mathcal{M}_{2}$ [15]. The operation \# is both commutative and associative. This latter property implies that multi-wormhole solutions of Einstein's equation can be constructed unambiguously.

Let us end with a discussion (and some speculations on) the applications of our results. To be of interest, wormholes need only stay open long enough for the radiation to traverse the throat. For the class of solutions treated here, a time scale is set by the frequency of the radiation traversing the throat, $\Delta t \sim 1 / \nu$, and a length scale by $\lambda \sim a_{i} R_{i}$. This follows since our wormholes have zero-length throats, that is, a particle going down the wormhole mouth in one region comes out the other end instantaneously (see Fig.1). Of course, we do not claim all wormholes will be of this form, but this still serves as a useful estimate of the minimum wormhole lifetime needed to initiate thermal contact at a fixed frequency. With these reservations [17] we now procede with a purely illustrative example. We assume a number density $n\left(t_{P l}\right)$ of Planck-sized wormholes (of radius $l_{P l}$ ) at the Planck time [18], and we let $n\left(t_{P l}\right)=\gamma n_{P l}\left(\gamma=\right.$ const.) [19] where $n_{P l}=\left(l_{P l}\right)^{-3}$ is the Planck number density. At some later time $t$, the wormhole number density is $n(t)=\gamma n_{P l}\left(R_{P l} / R(t)\right)^{3}$. The volume of space filled by the interior of wormhole mouths (volume filling factor) is

$$
\phi(t)=\frac{n(t)}{n_{P l}}\left(\frac{l(t)}{l_{P l}}\right)^{3} .
$$

For comoving wormholes $\phi(t)=\gamma$, as expected, but let us concentrate on dynamic $p=1 / 2$ 
wormholes where $l=a R$, for which one finds $\phi(t)=\gamma\left(R(t) / R_{P l}\right)^{3}$. Now for example, the average particle in the cosmic soup will have traversed at least one wormhole by time $t$ if $\gamma>\left(R(t) / R_{P l}\right)^{3}$. For example, by the GUT time, $t_{G U T} \sim 10^{-34} \mathrm{sec}$, a $\gamma \geq 10^{-21}$ allows thermalization. Thermalization at even the high $\nu$ end of the spectrum can (small wormholes only) eventually lead to thermal equilibrium at a later time for causally disconnected regions. This in turn could provide a wormhole solution to the horizon problem. It is rather natural to have an inflationary era in the early Universe. There may also be a cosmic wormhole era in the early Universe. However, to compete with inflation, a wormhole cosmology needs to address a list of other cosmological problems including flatness, monopoles (this may be solved directly in certain particle physics models) and density fluctuations. All these need further study.

TWK thanks the Institute of Theoretical Physics at Santa Barbara for hospitality while this work was in progress. This work was supported by the Department of Energy under Grant No. DE-FG05-85ER40226, and by the National Science Foundation under Grant No. PHY89-04035.

- 1. The status of early CMB measurements is reported in After the First Three Minutes, edited by S.S. Holt, C.L. Bennett and V. Trimble (American Institute of Physics, 1991). A dipole anisotropy has been previously seen in the COBE data, and recently evidence for higher multipole moments at the level of a few parts in $10^{5}$ has been reported in: "Interpretation of the CMB Anisotropy Detected by the COBE DMR," E. L. Wright et al., COBE preprint Apr. (1992), and "Structure in the COBE DMR First Year Maps", G. F. Smoot et al., COBE preprint Apr. (1992).

- 2. For a review of the standard big bang model, see, e.g., E.W. Kolb and M.S. Turner, The Early Universe, (Addison-Wesley, New York, 1990).

- 3. A.H. Guth, Phys. Rev. D23, 347 (1981); A.D. Linde, Phys. Lett. B108, 389 (1982); A. Albrecht and P.J. Steinhardt, Phys. Rev. Lett. 48, 1220 (1982). For a review of inflationary cosmology, see e.g., Ref. [2], Chap. 8; K. A. Olive, Phys. Rep. 
190, 307 (1990), and references therein.

- 4. The construction we employ here has been used to study wormholes in Minkowski, Schwarzschild and Nordström-Reissner backgrounds by M. Visser, Phys. Lett. B242, 24 (1990); Nucl. Phys. B 328, 203 (1989).

- 5. The scale factor $R$ is dimensionless; the curvature constant $\kappa=-1,0$ or 1 , corresponding to negative, flat or positively curved spatial sections, respectively.

- 6. W. Israel, N. Cimento 44B, 1 (1966); 48B, 463 (1967); C.W. Misner, K.S. Thorne and J.A. Wheeler, Gravitation, (Freeman, San Francisco, 1973), Chap 21.13.

- 7. The metric on the throat world-volume, ${ }^{(3)} g_{i j}=\operatorname{diag}\left(g_{\tau \tau}, g_{\theta \theta}, g_{\phi \phi}\right)$, has components $g_{\tau \tau}=-1, g_{\theta \theta}=a^{2}(\tau) R^{2}(t)$ and $g_{\phi \phi}=\sin ^{2} \theta g_{\theta \theta}$.

- 8. M.S. Morris and K.S. Thorne, Am. J. Phys. 56, 395 (1988); M. Morris, K.S. Thorne and U. Yurtsever, Phys. Rev. Lett. 61, 1446 (1988).

- 9. N.D. Birrell and P.C.W. Davies, Quantum Fields in Curved Space, (Cambridge U.P., Cambridge, 1982).

- 10. D. Hochberg and T.W. Kephart, Phys. Lett. B268, 377 (1991).

- 11. D. Hochberg and T.W. Kephart, VAND-TH-92-3, March 1992.

- 12. A quantum mechanical analysis of Minkowski signature wormholes in static background spacetimes has been carried out by M. Visser, Phys. Rev. D43, 402 (1991).

- 13. Though we adhere to $|\beta| \leq 1$, this may not be required since the matter corresponding to the negative energy density on the wormhole throat may behave tachyonically.

- 14. The scale factor for a radiation dominated Universe is $R(t)=\left(32 \pi G \rho_{\text {rad }} / 3\right)^{1 / 4} t^{1 / 2}$, where $\rho_{\text {rad }}=4.5 \times 10^{-34} \mathrm{gm} / \mathrm{cm}^{3}$ is the present radiation energy density.

- 15. See, e.g., W.S. Massey, Algebraic Topology, (Springer, Berlin, 1977). Our wormholes result from the trivial homeomorphism, $h \equiv$ identity.

- 16. D. Hochberg, Phys. Lett. B251, 349 (1990). 
- 17. It has been suggested that wormholes may form time machines (see second paper in Ref. [8]); if this were the case, the time travel aspects of wormholes could drive the Universe away from thermalization. However, based on quantum mechanical arguments, Hawking has argued (Phys. Rev. D46, 603 (1992)) and Visser has given a partial proof (WASH-U-HEP-92-70, Feb. 1992) that wormholes cannot be used for time-travel. Hence we assume travel through these wormholes always leads the Universe towards equilibrium.

- 18. The weak energy condition (WEC) is a classical conjecture that requires the energy density at each point in spacetime to be nonnegative. For an energy fluctuation $\Delta E \sim\left|\sigma a^{2} R^{2}\right|$, we find that Planck-sized wormholes of age $\Delta t \sim t_{P l}$ only violate the WEC at the quantum fluctuation level $\Delta E \Delta t \sim \hbar$, and so these wormholes cannot be ruled out by the WEC arguments. A longer-lived wormhole (say, a growing $p=1 / 2$ wormhole) will soon have $\Delta E \Delta t \geq \hbar$. Hence if the WEC is indeed applicable here, it will be violated, apparently implying the wormhole's eventual recollapse. We as yet do not know how to make these observations more quantitative.

- 19. Constant $\gamma$ implies the total number of wormholes is constant. This need not be the case in general, and we expect $\gamma=\gamma(t)$. 


\section{FIGURE CAPTION}

Figure 1. A spacetime diagram representation of a Friedmann cosmology with flat $\left(E^{3}\right)$ spatial sections, with the past lightcone for an observer at $A$. Radiation received at $A$ from opposite directions was emitted from $B$ and $C$. A wormhole connects events in the past lightcones of the otherwise spatially seperated points $B$ and $C$. A photon is shown going down the wormhole in $B$ 's past and exiting the wormhole in $C$ 's past. 